\title{
L'avenir de la vie et de la biodiversité
}

\section{Jean Martin}

Dr méd., membre de la rédaction

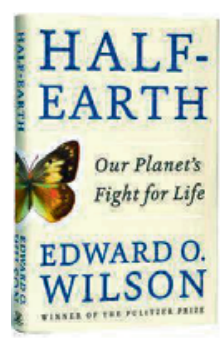

Edward O. Wilson

Half-Earth

Our Planet's Fight for Life

New York: Liveright Publishing Corporation; 2016.

259 pages. $33.90 \mathrm{CHF}$.

ISBN 978-1-63149-082-8

Edward O. Wilson (1929) est un biologiste américain, enseignant à Harvard, fondateur de la sociobiologie et un des pères de la discipline «biodiversité». Half-Earth est le troisième livre d'une trilogie consacrée à l'état de la planète, en particulier face aux risques d'extinction massive d'espèces.

«Pour la première fois dans l'histoire, la conviction se fait que nous jouons une dernière partie (endgame) au niveau mondial. Nous nous agitons, menés [au plan politique] de manière consternante, sans autre but que la croissance économique et la consommation. Notre impact sur la biosphère est partout négatif [...]. Notre espèce est devenue l'architecte de l'ère anthropocène avec des conséquences qui affecteront toute vie loin dans le futur géologique. $»^{1}$

Trois parties au livre: le Problème, le Monde vivant réel, la Solution. La première est consacrée à la biosphère, à la biodiversité et aux interactions entre espèces. Pages passionnantes fourmillant d'illustrations sur le devenir (en particulier la disparition!) de nombreuses espèces d'animaux. La deuxième s'attache à la nature dans diverses dimensions, à ses habitants et à sa protection (conservation), avec un historique et beaucoup d'exemples - Wilson lui-même s'est notamment impliqué en faveur du parc national de Gorongosa au Mozambique. Il a procédé à une enquête parmi d'éminents collègues pour lister les endroits les plus importants/riches de la biosphère, sur les différents continents. La troisième partie présente des pistes de résolution des problèmes.
Le constat: «Nous sommes sur un chemin de destruction [...]. La vie sur Terre est dans un goulet d'étranglement dû à la croissance démographique, à la raréfaction des ressources et à l'extinction des espèces. Or, nous sommes l'esprit (mind) et les intendants (stewards) du monde vivant et devons nous considérer comme responsables de le sauver.» Rappelant comme Michel Serres [1] qu'il importe d'intégrer une vue longue de l'évolution: «Des millions d'années sont encodées dans nos gènes. L'histoire considérée sans les régions sauvages vierges n'est pas l'histoire.» Et aussi: «La biosphère ne nous appartient pas; c'est nous qui lui appartenons.» «Nous avons besoin d'une compréhension beaucoup plus profonde de la vie sur terre que ce que les sciences humaines et la science ont offert jusqu'ici [...]. Un changement d'orientation et un engagement fort pour les autres êtres vivants sont nécessaires.»

«La science montre que notre système sensoriel et notre raisonnement moral ne parviennent pas à répondre aux enjeux actuels. Nous sommes remarquablement adaptés au monde d'hier, mais loin de l'être pour ce qui arrive. L'humanité doit apprendre de et sur la biodiversité et agir rapidement pour la protéger.»

Sa solution: «Ma proposition de Half-Earth offre une solution d'urgence à la dimension du problème; c'est seulement en mettant de côté [à l'écart de l'action humaine, de manière inviolable], 'en réserve', la moitié de la planète, ou plus, que nous pouvons sauver la partie vivante de notre milieu et parvenir à la stabilisation indispensable à la survie.» Il est possible de le faire, affirme-t-il. D'une manière qui peut surprendre venant de quelqu'un si proche de la nature - et son avocat, Wilson est convaincu que les avancées scientifiques apporteront beaucoup pour permettre une/des vies acceptables sur la Terre. «Les 'bnr' (biologie - y compris neurosciences, nanotechnologie, robotique) ont le potentiel soit de soutenir la biodiversité, soit de la détruire. Je crois qu'elles vont la soutenir, [notamment] en favorisant les énergies renouvelables et en diminuant le besoin et même le désir de voyager. Nous réduirons notre empreinte écologique grâce à elles.»

Ainsi, Wilson voit beaucoup de potentiel dans l'intelligence artificielle et la "whole brain emulation" (construction de logiciel capable de modéliser le fonctionnement complet du cerveau humain - avec même le but à terme «d'expliquer notre conscience»). 